\title{
Design of global positioning system (GPS) networks using different artificial intelligence techniques
}

\author{
Doaa. S. Odam*, Mohamed. I. Doma**, Hossam. I. Fawzy ${ }^{* * *}$, Ahmed A. Sedeek ${ }^{* * *}$ and Magda. H. \\ Farhan ${ }^{* * * *}$ \\ "Civil Department, Higher Institute of Engineering and Technology, Kafr El-Sheikh, Egypt \\ ${ }^{* *}$ Civil Department, Faculty of Engineering, Menoufia University, Egypt \\ ${ }^{* * *}$ Civil Department, Faculty of Engineering, Kafr El-Sheikh University, Egypt \\ ${ }^{* * * *}$ Civil Department, EL Behira Higher Institute of Engineering and Technology, El Behira, Egypt \\ *Corresponding Author: doaa.pg_0026@eng.kfs.edu.eg
}

Submitted: 18-05-2020

Revised: 09-07-2021

Accepted: 24-07-2021

\begin{abstract}
The selection of optimal GPS baselines can be realized by solving the geodetic second-order design (SOD) problem. Basically, there are two techniques to be used for selecting optimal baselines in GPS network, namely, traditional techniques and artificial techniques. Traditional techniques include the method of trial and error and the analytical method, while artificial methods include both local and global optimization techniques. The global optimization techniques, such as Genetic Algorithms (GAs), Simulated Annealing (SA) method, Particle Swarm Optimization (PSO) Algorithm, and Butterfly Optimization Algorithm (BOA), have been used recently in geodesy. In the current study, BOA has been used for the selection of the optimal GPS baselines to be measured in the field that will meet the postulated criterion matrix, at a reasonable cost. It has been tested on a GPS network. The BOA is already designed, and it determined the number of baselines that would be observed because of obtaining high accuracy. The results showed that the BOA method was more efficient than the traditional ones by $19.2 \%$. It was better than the artificial methods in terms of length as it enhanced SA method by $21.7 \%$ and PSO method by $4.6 \%$. Consequently, the use of the BOA is proven to be more effective and applicable.
\end{abstract}

Keywords: GPS networks; Artificial Intelligence; Butterfly Optimization Algorithm (BOA); Secondorder design problem.

\section{INTRODUCTION}

Global Positioning System (GPS) is mainly used to create a special precise geodetic network to monitor deformations and crustal movements (Dwivedi and Dikshit, 2013). GPS is an advanced technology, which plays a vital role in our daily life, whereas GPS applications are becoming increasingly important in our dynamic world (Hoque, 2016). Optimization design and optimal geodetic network play a key role in many geodetic applications (Doma, 2013), whereas the geodetic network quality is characterized by its reliability, cost, and precision. The main objective of designing optimal network and optimal observational plan is to provide high reliability and excellent precision, at minimum cost (Amiri-Simkooei et al., 2012). The optimal design of GPS networks can be attained by following the traditional approach to optimize the terrestrial geodetic networks, as follows (Grafarend, 1974): Zero-order design (ZOD), Optimum datum definition, and First-order design (FOD). Design an optimal network configuration, Second-order design (SOD): Specification of observation weights, Third-order design (THOD): Development of the existing network by removing and adding observations.

Among the four abovementioned orders of design, the SOD problem is generally discussed in literature and shown in practice. The main problem of the optimization GPS network and the traditional SOD problem is the choice of the most optimal baselines to be observed in the field (Dwivedi and Dikshit, 2013). The main objective of SOD is to determine the best economic survey movement that meets the required network optimization criteria. 
The SOD identifies the observations to be made and their corresponding accuracy. Moreover, it facilitates the choice of the proper instrument and observation procedures (Yetkin et al., 2009). The technique of optimization provides the optimal weights of observation, which can then be classified into zero, small, or optimized weights. The optimized weights can also be much less than the initial weights; they are then modified by their corresponding initial weights. Baselines that achieve zero or small weight signify that such baselines must not be observed (Doma, 2013). Artificial intelligence techniques are used to analyze large amounts of data to establish an ideal GPS network design based on geometrical and financial constraints. Approaches and algorithms such as large networks and logistics include local and global optimization, which can meet the needs of GPS networking (Kota, 2018; Habeeb, 2018). Achieving optimization allows for the selection of suitable GPS baselines, and it can be realized by solving the geodetic second-order design (SOD) problem. Basically, there are two techniques to be used for selecting optimal baselines in GPS network, namely, traditional techniques and artificial techniques. Traditional techniques use trial and error and the analytical methods (Alizadeh-Khameneh, 2017). Yet, the artificial techniques encompass local optimization and global optimization techniques shown in figure (1). Lately, global optimization techniques like GA method, SA method, PSO algorithm, and BOA method have been recently introduced to geodesy (Fig (1)) (Doma et al., 2013).

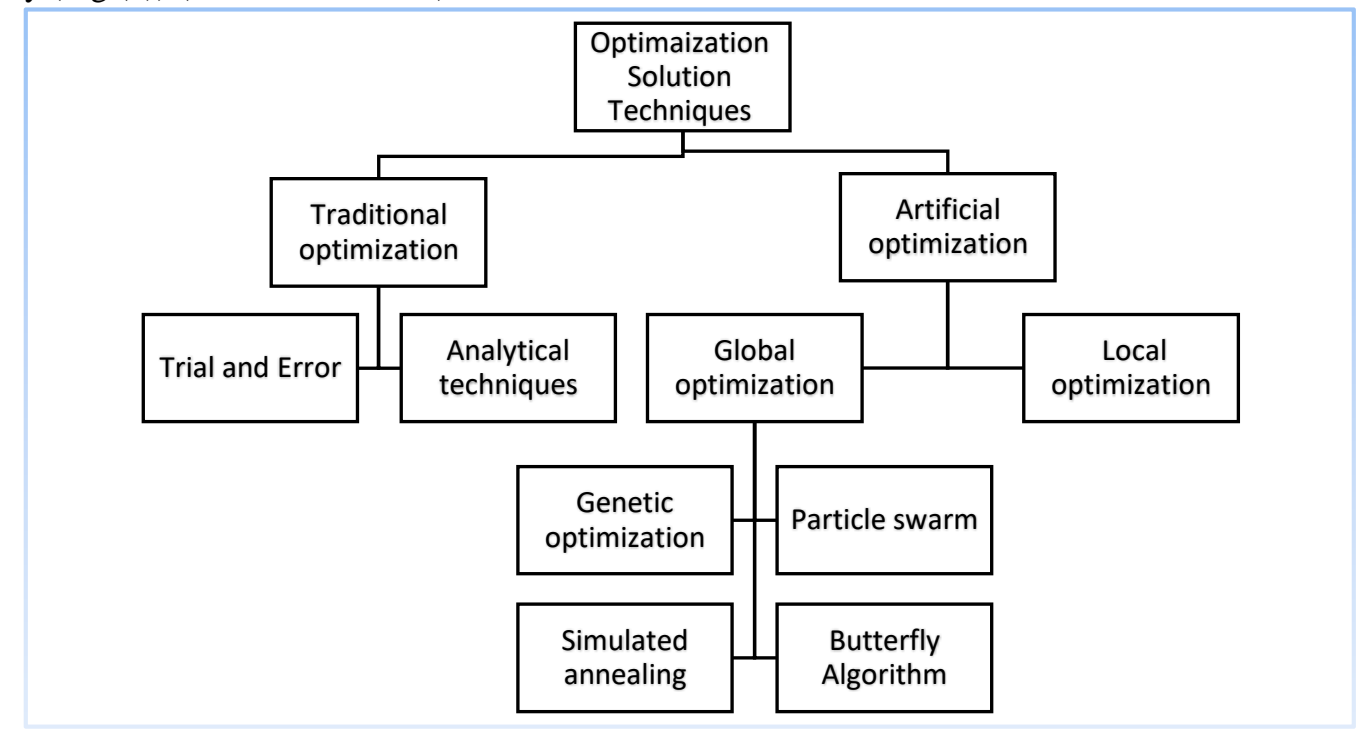

Figure 1. Optimization Solution Techniques.

\section{LITERATURE REVIEW}

In traditional techniques, Kuang (1996) used the same GPS network as in our work. After decreasing (2.00 $\mathrm{mm}$ ) from the precision of the network, a total of 73 baselines were removed (Kuang, 1996). In artificial techniques, SA method was applied to this network, and a total of 79 baselines were removed (Faried, Doma 2013). PSO method was applied to this network, and a total of 87 baselines were removed (Doma, 2013). The optimization of a geodetic network is the act of designing a specific step, so that the end product matches the expected (optimal) design requirements. The purpose of optimization is to find a model that meets the adopted reference system's requirements/criteria ( Postek, 2021).

\section{OBJECTIVES}

The main objectives are as follows:

1. Implementation of a monitoring plan to improve GPS networks using Artificial intelligence-based BOA.

2. Comparison of Artificial intelligence-based BOA with traditional methods and artificial methods such as SA and PSO to choose the optimal technique to improve the GPS network.

\section{MATHEMATICAL EQUATION OF THE GPS NETWORK PROBLEM}

The most accurate GPS-based positions are acquired when all satellites are observed as long as possible, and all baselines are measured and recorded in the network. This is extremely difficult in practice because of payments 
and time. Thus, optimum survey design must be done to get the prescribed design criteria at minimum cost. In optimization problems, $\mathrm{P}$ is the matrix of observation weights, and $\mathrm{A}$ is the matrix that acts as the geometry of the network. In case the two matrices are known, the covariance matrix of the unknowns is given by (Doma and Sedeek, 2012; Doma, 2013)

$$
Q_{x}=\left(A^{T} P A\right)^{-1}
$$

As generally stated, SOD aims to determine the weights of P (perhaps only for some "baselines" of observations) that create the resulting cofactor matrix. The cofactor matrix could be in any required form. For example, it might follow the matrix criterion of Baarda (Baarda, 1981) that leads to the regions of absolute confidence and relative circular shape, or it can simply be a diagonal matrix (although it is not possible in practice). Anyways, let us determine the degree of approximation to the required solution through applying the Frobenius norm - that provides the distance of Euclidean between matrices (Doma and Elshoney, 2011). Hence, the global problem of optimization is then pointed out as the problem of calculating the weights contained in $\mathrm{P}$ (Equation (1)) for which the following global minimum is obtained (Kuang, 1991 and 1996 ; Yetkin et al., 2008 and 2011 ; Doma, 2013):

$$
\min \left\|Q_{f}-Q_{x}\right\|=\min \sqrt{\sum_{i} \sum_{j}\left(\left(Q_{f}\right)_{i j}-\left(Q_{x}\right)_{i j}\right)^{2}}
$$

As a new strategy, some constraints can be put on the weights to be obtained in the present study. Such constraints include at least the need for some of the weights to be negative in order to delete them from the final observational plan. The solution to Equation (2) will always exist, but obviously, the attained degree of approximation to the desired matrix will be prticularly dependent on the problem itself.

A MATLAB code was adapted using BOA techniques to optimize a presolved example to asses the BOA as an artifical intelligant technique in solving SOD.

\section{BUTTERFLY OPTIMIZATION ALGORITHM}

Butterfly Optimization Algorithm (BOA) is a new nature inspired algorithm, which mimics the pattern of butterflies' food searching strategy (Arora and Singh, 2015). Butterflies act as the search agents used by the BOA to implement optimization. In BOA, it is assumed that a butterfly will release scent with different intensities according to the butterfly fitness. The scent will spread, and other butterflies can feel it (Arora and Singh, 2017). The stages of BOA are as follows: initialization stage, iteration stage, and final stage.

During each iteration process, the butterflies in the solution space take new positions. Then, their corresponding fitness values are calculated by the algorithm according to the following equation (Arora and Singh, 2015 and 2019):

$$
\boldsymbol{f}=\boldsymbol{c} I^{a}
$$

where $(f)$ is the amount of the scent received by other butterflies, (c) refers to the sensory modality, (I) refers to the stimulus intensity, and (a) refers to the power according to modality, which measures different degrees of absorption. The algorithm is divided into two key steps: local search phase and global search phase. At the stage of global search, take the butterfly step towards the fittest butterfly /solution g, which can be formulated as follows (Arora and Singh, 2015 \& 2019):

$$
x_{i}^{t+1}=x_{i}^{t}+\left(r^{2} \times g^{*}-x_{i}^{t}\right) \times f_{i}
$$

where $\left(x_{i}{ }^{t}\right)$ is the solution vector. $\left(x_{i}\right)$ is the butterfly in iteration number $(\mathrm{t}) .\left(g^{*}\right)$ introduces the best currently found solution in the current stage. The scent of a butterfly is shown by $\left(f_{i}\right)$. (r) is a random number in $[0,1]$. The local search phase can be summed up as follows:

$$
x_{i}^{t+1}=x_{i}^{t}+\left(r^{2} \times x_{k}^{t}-x_{j}^{t}\right) \times f_{i}
$$

where $\left(x_{j}{ }^{t}\right)$ and $\left(x_{k}{ }^{t}\right)$ are $\mathrm{j}^{\text {th }}$ and $\mathrm{k}^{\text {th }}$ butterflies chosen randomly from the solution space. If $x_{j}{ }^{t}$ and $x_{k}{ }^{t}$ belong to the same subgroup, $(\mathrm{r})$ is the random number in $[0,1]$. The local and global scales exist when the butterflies search 
for mating partners and foods. Therefore, a switch possibility (p) is used in BOA to switch between joint global search and intensive local search. In Figure (2), the flow chart describes the optimization stages of the BOA and the proposed approach. The computational stages of the proposed BOA are presented below, and its pseudocode is described in Algorithm 1 in Figure (3) (Arora and Singh, 2015 \& 2019).

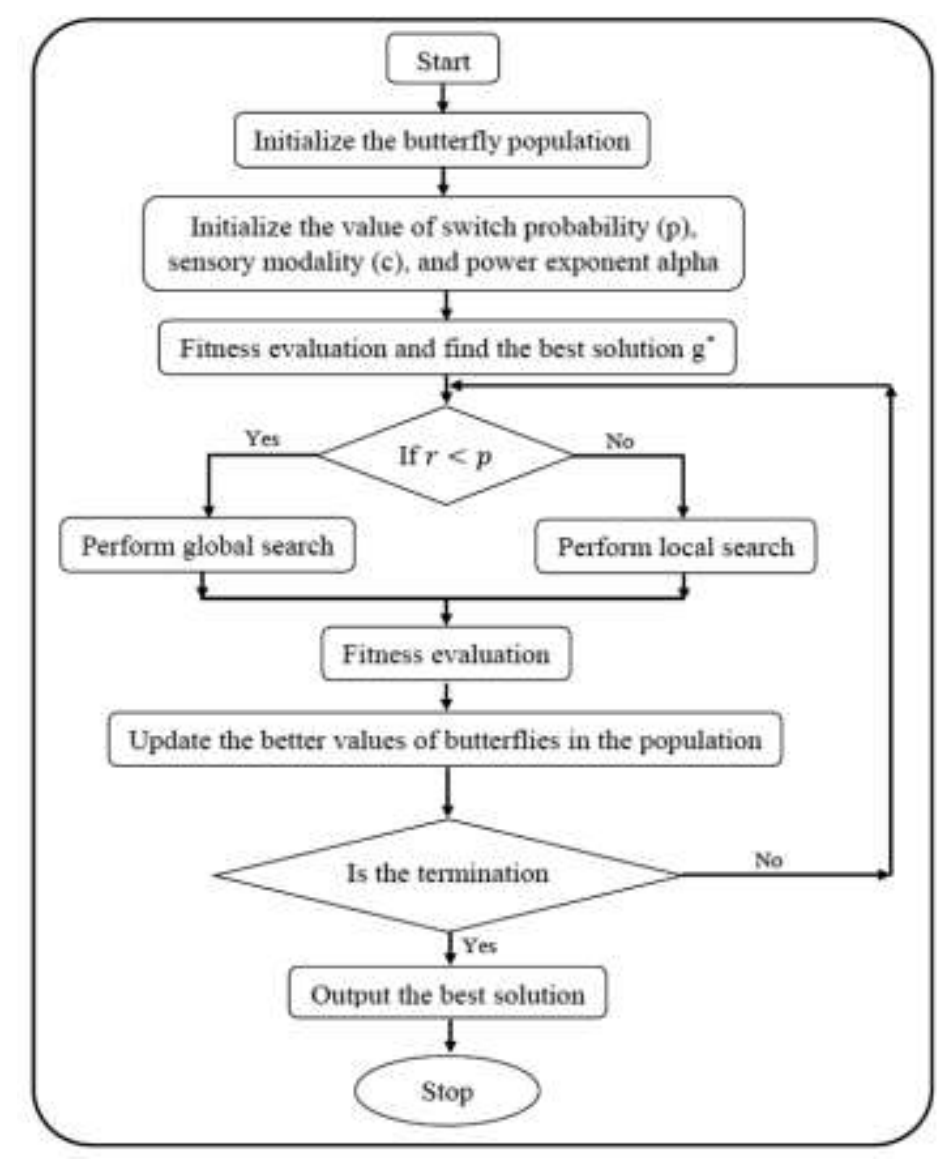

Figure 2. Algorithm 1 Butterfly optimization algorithm.

\begin{tabular}{|ll|}
\hline Algorithm Butterfly optimization algorithm: \\
2: $\quad$ Objective function $f(x), x=\left(x_{1}, x_{2}, x_{3}, \ldots \ldots x_{\text {dim }}\right)$, dim $=$ no. of dimensions \\
3: $\quad$ Stimulus Intensity $I_{\mathrm{i}}, x_{i}$ is determined by $f\left(x_{i}\right)$ \\
4: $\quad$ Define c, a and $\mathrm{b}$ \\
5: $\quad$ While stopping criteria not met do \\
6: $\quad$ for each butterfly $\boldsymbol{b} \boldsymbol{f}$ in population do \\
7: $\quad$ Calculate fragrance for $\boldsymbol{b} \boldsymbol{f}$ using Eq. (5) \\
8: $\quad$ end for \\
9: $\quad$ Find the best $\mathrm{b} \boldsymbol{f}$ \\
10: $\quad$ for each butterfly $\boldsymbol{b} \boldsymbol{f}$ in population do
\end{tabular}




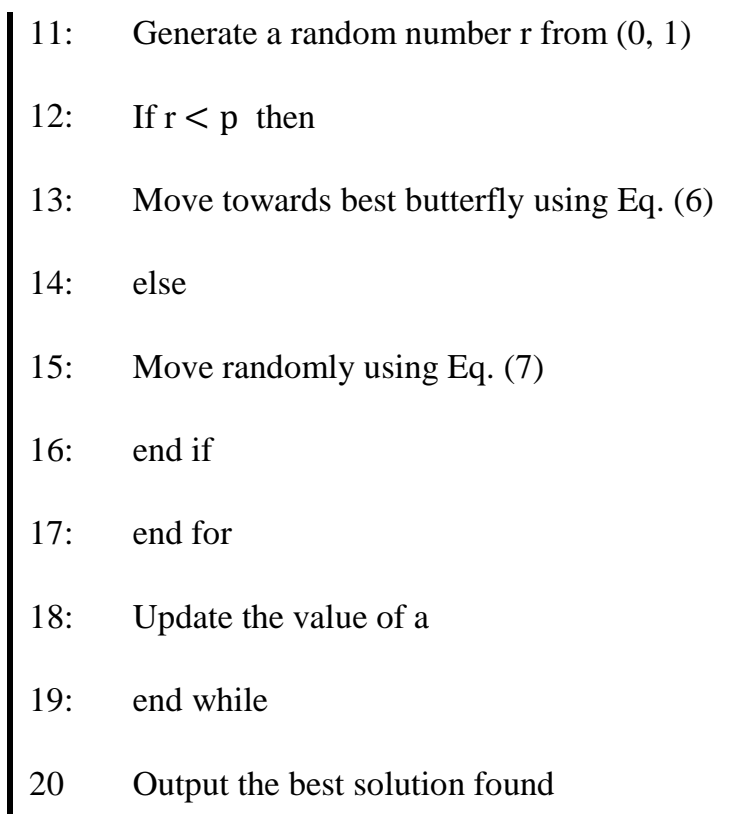

Figure 3. Algorithm butterfly optimization algorithm.

\section{CASE STUDY}

Kuang (1996) used a network consisting of 18 stations, which were observed using the GPS relative positioning technique and 153 baselines. The lengths of the baselines ranged from (2.8) to (36) km, and their total length equaled $2386.87 \mathrm{~km}$, as shown in Fig. (4). The selected GPS receivers assumed that the precision of the baselines $\left(\sigma_{s}\right)$ could be determined via the following equation (Doma, 2013):

$$
\sigma_{s}^{2}=(2)^{2} m m^{2}+(1 \text { ppm.S })^{2}
$$

S: the baseline length $\sigma_{s}$ : The precision of baselines

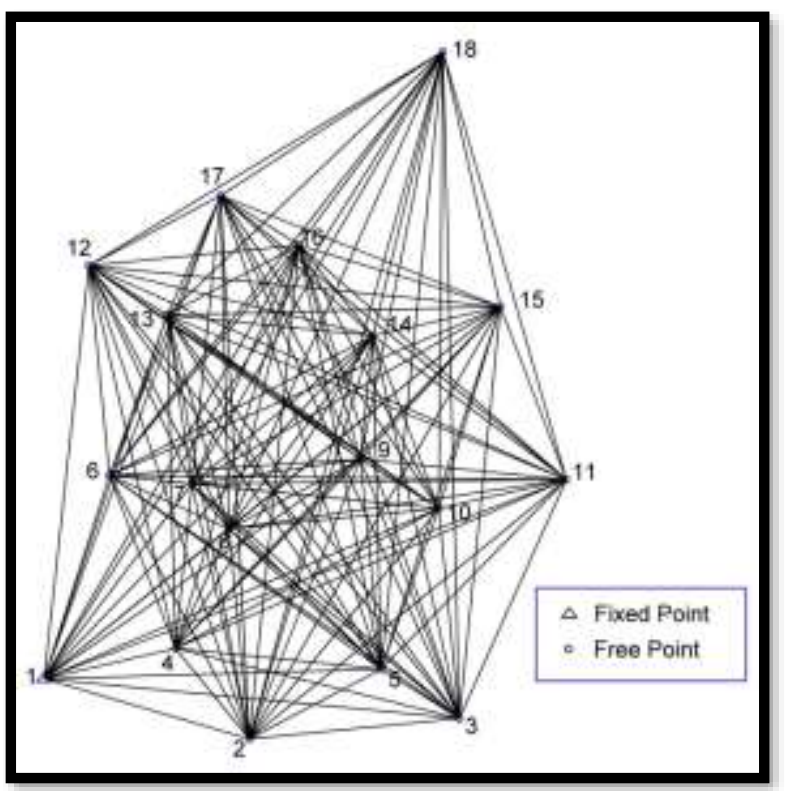

Figure 4. All possible combinations of baselines.

Depending on the proposed configuration of the land, the maximum number of semi-independent baselines is 153. If the measurement of the 153 baselines is observed precisely, as shown in Eq. (6), the standard deviations 
of the coordinate elements of every station (given that station (1) is chosen as the fixed datum point) are recorded below $\left(\sigma_{o}\right)$. In this network, this is the maximum accuracy that can be achieved if all possible baselines are measured. Kuang (1996) suggested that the optimization modeling has been executed using diagonal matrix as its criterion matrix (Doma, 2013):

$$
Q_{f}=\operatorname{diag}\left\{\begin{array}{llllll}
0.0 & 0.0 & 0.0 & \sigma_{2 x}^{2} \sigma_{2 y}^{2} \sigma_{2 z}^{2} \cdots \cdots & \sigma_{18 x}^{2} \sigma_{18 y}^{2} \sigma_{18 z}^{2}
\end{array}\right\}
$$

where

$$
\sigma_{i j}^{2}=\left(\sigma_{o i j}+2.0\right)^{2}\left(m m^{2}\right)(\mathbf{i}=2,3, \ldots \ldots \ldots, 18 ; \mathbf{j}=\mathbf{x}, \mathbf{y}, \mathbf{z}
$$

$\sigma_{o i j}$ are the minimum possible standard deviations of the coordinate elements of the network stations.

Taking into consideration the maximum achievable weights obtained from the maximum achievable precision expressed by Equation (6), these weights are considered the initial values of observations for the two methods "traditional method (according to Kuang 1996)" and "the proposed method" (BOA method). In Kuang's model (1996), a total of 73 baselines were removed after decreasing the precision of the network by $2.0 \mathrm{~mm}$ (saving $47.7 \%$ of the fieldwork). The standard deviations of the station coordinates, as drawn from the optimized observational scheme, are listed under $\sigma_{p}$. follows:

The basic steps for applying the BOA technique to procedure a SOD expressed in equation (2) are given as

1- Determine an initial cofactor matrix $\left(Q_{x}\right)$ : from the calculation of $\mathrm{P}$ and $\mathrm{A}$ matrices, calculate the cofactor matrix expressed in Eq. (1) for all possible baselines.

2- Explain the search area: use the minimum and maximum weights to explain the search area, that is, how butterfly positions are rated at minimum and maximum weights. Minimum weights $=0.0$, while maximum weights are determined on the basis of the precision criterion, as explained by Eq. (6).

3- Explain the model of required optimization: determine $\left(Q_{f}\right)$, as explained in Eq. (7).

4- Run (BOA) code: (see the code of MATLAB).

Using the Pc-MATLAB version with a personal computer and the developed mathematical model, this program has been written to calculate the optimal baselines to be selected for GPS survey planning; see Fig. (5).

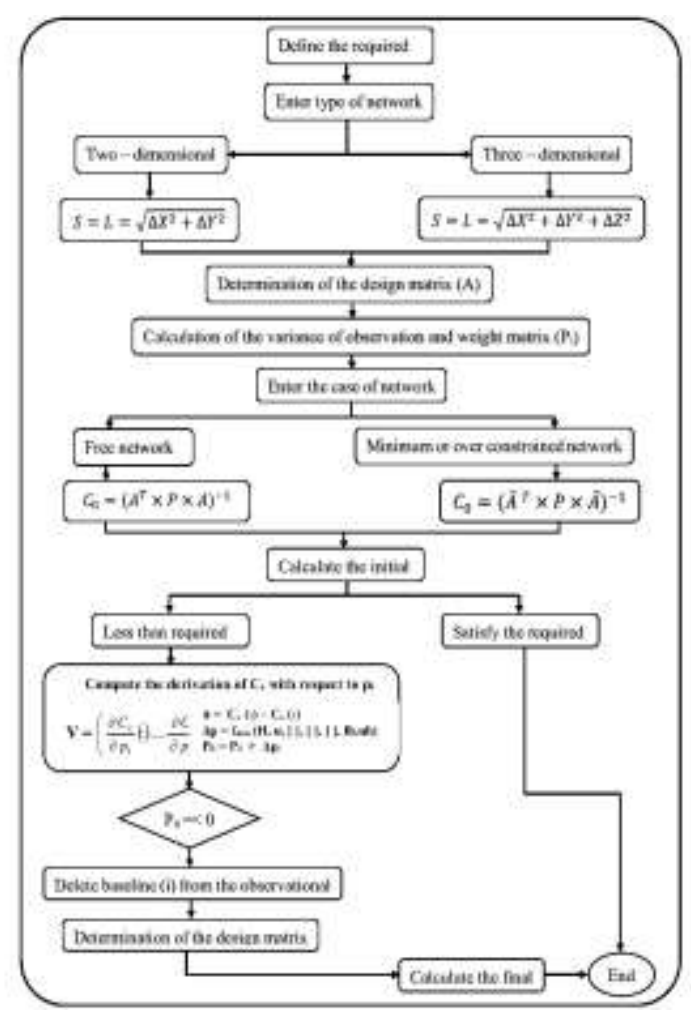


Figure 5. Flowchart of the program.

In the current study, if we allowed for a decrease of $2.0 \mathrm{~mm}$ in the precision of the network and used the proposed BOA method to obtain the zero or insignificant weights, a total of 87 baselines should be removed, which means saving $56.9 \%$ of the fieldwork. The standard deviations of the station coordinates, as drawn from the optimized observational chart, are listed under $\sigma_{p}$. Column 13 of Table (1) lists the differences between $\sigma_{o}$ and $\sigma_{p}$; all the standard deviations of the coordinate elements obtained from the three methods are less than and close to the required value shown in Figure (6).

Table 1. A comparison of the standard deviations of the coordinates before and after optimization. (using traditional method and artificial method) ("Kuang, 1996", SA, PSO, and BOA method).

\begin{tabular}{|c|c|c|c|c|c|c|c|c|c|c|c|c|}
\hline \multirow[t]{2}{*}{ Point } & \multicolumn{4}{|c|}{ (Kuang, 1996) } & \multicolumn{2}{|c|}{ (SA method) } & \multicolumn{2}{|c|}{ (PSO method) } & \multicolumn{4}{|c|}{ (BOA method) } \\
\hline & $\sigma_{0}$ & $\sigma_{\mathrm{P}}$ & $\sigma_{0}-\sigma_{P}$ & $\sigma_{0}$ & $\sigma_{\mathrm{P}}$ & $\sigma_{0}-\sigma_{P}$ & $\sigma_{0}$ & $\sigma_{\mathrm{P}}$ & $\sigma_{0}-\sigma_{P}$ & $\sigma_{0}$ & $\sigma_{\mathrm{P}}$ & $\sigma_{0}-\sigma_{P}$ \\
\hline 1 & 0.00 & 0.00 & 0.00 & 0.00 & 0.00 & 0.00 & 0.00 & 0.00 & 0.00 & 0.00 & 0.00 & 0.00 \\
\hline 2 & 4.45 & 6.34 & 1.89 & 4.45 & 6.46 & 2.01 & 4.45 & 6.43 & 1.98 & 4.45 & 6.13 & 1.68 \\
\hline 3 & 4.81 & 6.47 & 1.66 & 4.81 & 6.82 & 2.01 & 4.81 & 6.44 & 1.63 & 4.81 & 6.79 & 1.98 \\
\hline 4 & 4.01 & 5.57 & 1.55 & 4.01 & 6.02 & 2.01 & 4.01 & 5.69 & 1.68 & 4.01 & 5.39 & 1.38 \\
\hline 5 & 4.49 & 6.31 & 1.82 & 4.49 & 6.50 & 2.01 & 4.49 & 6.04 & 1.55 & 4.49 & 6.46 & 1.97 \\
\hline 6 & 4.23 & 5.71 & 1.48 & 4.23 & 6.24 & 2.01 & 4.23 & 5.93 & 1.70 & 4.23 & 6.12 & 1.89 \\
\hline 7 & 4.06 & 5.69 & 1.63 & 4.06 & 6.07 & 2.01 & 4.06 & 5.81 & 1.75 & 4.06 & 6.01 & 1.95 \\
\hline 8 & 4.07 & 5.90 & 1.84 & 4.07 & 6.08 & 2.01 & 4.07 & 6.00 & 1.93 & 4.07 & 5.98 & 1.91 \\
\hline 9 & 4.32 & 6.03 & 1.71 & 4.32 & 6.36 & 2.01 & 4.32 & 6.26 & 1.94 & 4.32 & 5.90 & 1.58 \\
\hline 10 & 4.44 & 6.30 & 1.86 & 4.44 & 6.45 & 2.01 & 4.44 & 6.08 & 1.64 & 4.44 & 6.27 & 1.83 \\
\hline 11 & 4.96 & 6.97 & 2.01 & 4.96 & 6.98 & 2.02 & 4.96 & 6.96 & 2.00 & 4.96 & 6.96 & 2.00 \\
\hline 12 & 4.71 & 6.60 & 1.88 & 4.71 & 6.68 & 1.97 & 4.71 & 6.50 & 1.80 & 4.71 & 6.65 & 1.94 \\
\hline 13 & 4.44 & 6.23 & 1.78 & 4.44 & 6.44 & 2.00 & 4.44 & 6.15 & 1.71 & 4.44 & 6.43 & 1.99 \\
\hline 14 & 4.46 & 6.26 & 1.80 & 4.46 & 6.54 & 2.08 & 4.46 & 6.23 & 1.77 & 4.46 & 5.73 & 1.27 \\
\hline 15 & 4.88 & 6.84 & 1.96 & 4.88 & 6.90 & 2.02 & 4.88 & 6.90 & 2.02 & 4.88 & 6.85 & 1.97 \\
\hline 16 & 4.53 & 6.52 & 1.99 & 4.53 & 6.53 & 2.00 & 4.53 & 6.21 & 1.68 & 4.53 & 6.34 & 1.81 \\
\hline 17 & 4.66 & 6.52 & 1.86 & 4.66 & 6.70 & 2.04 & 4.66 & 6.30 & 1.64 & 4.66 & 6.48 & 1.82 \\
\hline 18 & 5.96 & 7.91 & 1.95 & 5.96 & 7.93 & 1.97 & 5.96 & 7.74 & 1.78 & 5.96 & 7.80 & 1.84 \\
\hline
\end{tabular}




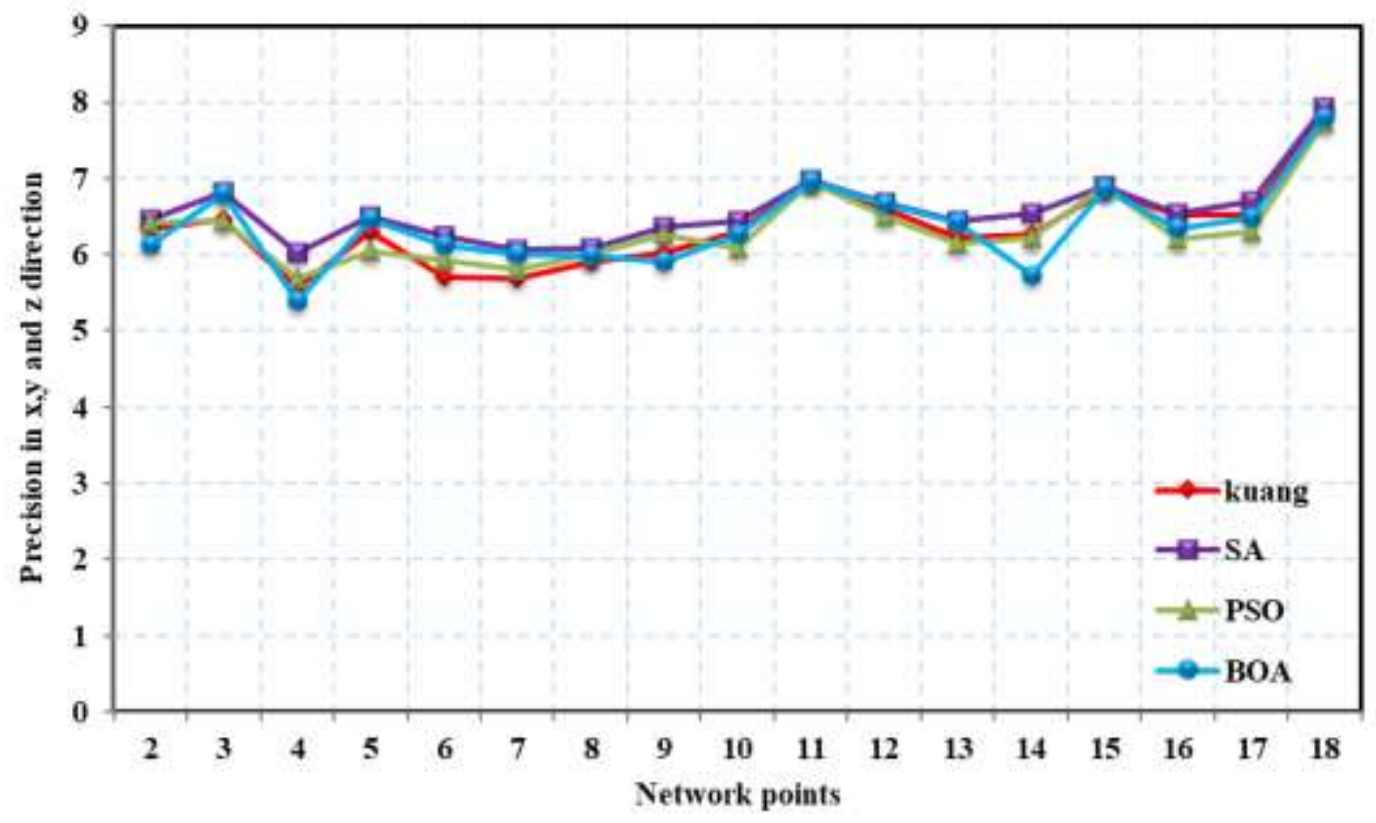

Figure 6. Standard deviations obtained through traditional and artificial intelligence techniques.

Figure (7) shows the optimized observation chart and the total length of the removed baselines equal 1382.85 $\mathrm{km}$, which means saving $57.9 \%$ (as shown in Table (2)).

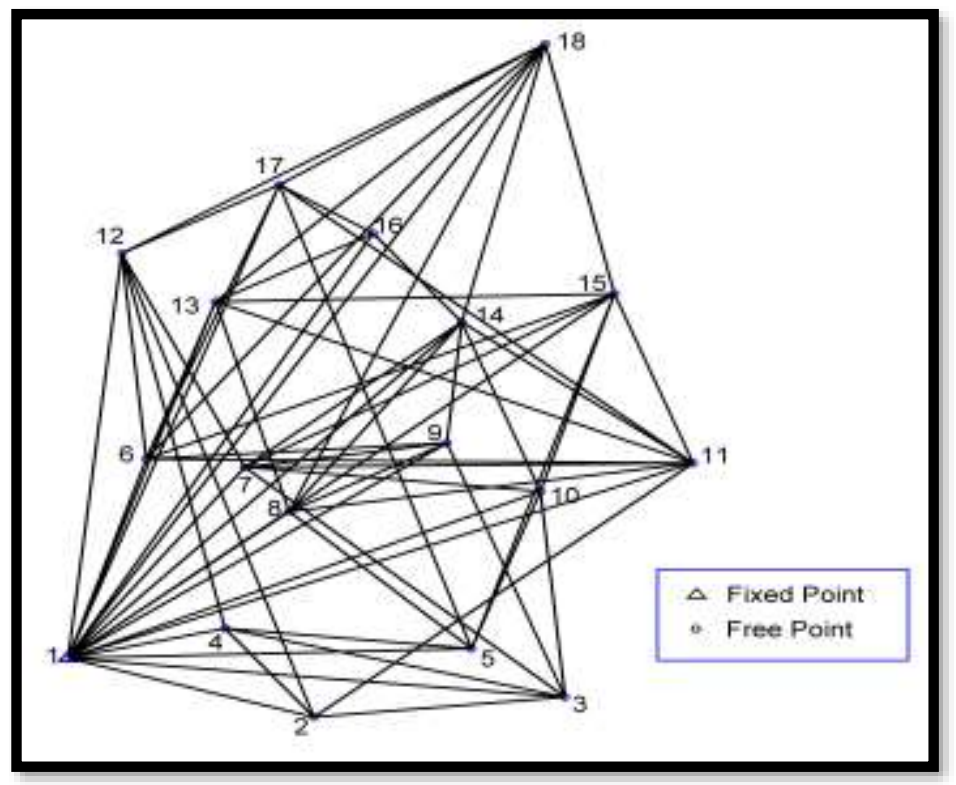

Figure 7. The final baselines after applying (BOA) optimization. 
Table 2. The baselines deleted due to optimization (using BOA method).

\begin{tabular}{|c|c|c|c|c|c|c|c|}
\hline \multirow{2}{*}{ No. } & \multicolumn{2}{|c|}{ Baseline } & \multirow{2}{*}{ Length (km) } & \multirow{2}{*}{ No. } & \multicolumn{2}{|c|}{ Baseline } & \multirow{2}{*}{ Length $(\mathrm{km})$} \\
\hline & From & To & & & From & To & \\
\hline 1 & 2 & 5 & 7.213 & 45 & 6 & 7 & 4.00 \\
\hline 2 & 2 & 6 & 14.42 & 46 & 6 & 8 & 6.324 \\
\hline 3 & 2 & 7 & 12.65 & 47 & 6 & 10 & 16.00 \\
\hline 4 & 2 & 8 & 10.20 & 48 & 6 & 14 & 14.42 \\
\hline 5 & 2 & 9 & 14.56 & 49 & 6 & 16 & 14.42 \\
\hline 6 & 2 & 10 & 14.42 & 50 & 7 & 8 & 2.83 \\
\hline 7 & 2 & 13 & 20.88 & 51 & 7 & 13 & 8.25 \\
\hline 8 & 2 & 14 & 20.40 & 52 & 7 & 16 & 12.65 \\
\hline 9 & 2 & 15 & 24.17 & 53 & 7 & 17 & 14.00 \\
\hline 10 & 2 & 16 & 24.00 & 54 & 7 & 18 & 24.17 \\
\hline 11 & 2 & 17 & 26.31 & 55 & 8 & 10 & 10.20 \\
\hline 12 & 2 & 18 & 34.52 & 56 & 8 & 12 & 14.42 \\
\hline 13 & 3 & 5 & 4.472 & 57 & 8 & 15 & 16.97 \\
\hline 14 & 3 & 6 & 20.59 & 58 & 8 & 16 & 14.14 \\
\hline 15 & 3 & 8 & 14.42 & 59 & 8 & 17 & 16.13 \\
\hline 16 & 3 & 11 & 12.65 & 60 & 9 & 10 & 4.47 \\
\hline 17 & 3 & 12 & 28.30 & 61 & 9 & 11 & 10.00 \\
\hline 18 & 3 & 13 & 24.09 & 62 & 9 & 12 & 16.13 \\
\hline 19 & 3 & 14 & 18.97 & 63 & 9 & 13 & 11.66 \\
\hline 20 & 3 & 15 & 20.00 & 64 & 9 & 15 & 10.00 \\
\hline 21 & 3 & 16 & 24.17 & 65 & 9 & 16 & 10.77 \\
\hline 22 & 3 & 17 & 27.79 & 66 & 9 & 17 & 14.43 \\
\hline 23 & 3 & 18 & 32.25 & 67 & 9 & 18 & 20.10 \\
\hline 24 & 4 & 6 & 8.944 & 68 & 10 & 11 & 6.324 \\
\hline 25 & 4 & 7 & 8.00 & 69 & 10 & 12 & 20.60 \\
\hline
\end{tabular}




\begin{tabular}{|c|c|c|c|c|c|c|c|}
\hline 26 & 4 & 8 & 6.324 & 70 & 10 & 13 & 16.13 \\
\hline 27 & 4 & 9 & 12.81 & 71 & 10 & 16 & 14.42 \\
\hline 28 & 4 & 10 & 14.42 & 72 & 10 & 17 & 18.44 \\
\hline 29 & 4 & 11 & 20.59 & 73 & 10 & 18 & 22.10 \\
\hline 30 & 4 & 13 & 16.12 & 74 & 11 & 12 & 25.30 \\
\hline 31 & 4 & 14 & 17.89 & 75 & 11 & 16 & 17.20 \\
\hline 32 & 4 & 15 & 22.80 & 76 & 11 & 18 & 21.54 \\
\hline 33 & 4 & 16 & 20.39 & 77 & 12 & 13 & 4.474 \\
\hline 34 & 4 & 17 & 22.00 & 78 & 12 & 14 & 14.15 \\
\hline 35 & 4 & 18 & 31.62 & 79 & 12 & 15 & 20.00 \\
\hline 36 & 5 & 6 & 16.13 & 80 & 12 & 16 & 10.20 \\
\hline 37 & 5 & 7 & 12.81 & 81 & 13 & 14 & 10.00 \\
\hline 38 & 5 & 9 & 10.20 & 82 & 13 & 17 & 6.324 \\
\hline 39 & 5 & 11 & 12.81 & 83 & 14 & 15 & 6.324 \\
\hline 40 & 5 & 12 & 24.09 & 84 & 14 & 17 & 10.00 \\
\hline 41 & 5 & 13 & 20.00 & 85 & 15 & 16 & 10.20 \\
\hline 42 & 5 & 14 & 16.13 & 86 & 15 & 17 & 14.56 \\
\hline 43 & 5 & 16 & 20.88 & 87 & 16 & 18 & 11.66 \\
\hline 44 & 5 & 18 & 30.00 & -- & -- & -- & -- \\
\hline
\end{tabular}

\section{DISCUSSION OF RESULTS}

We compared the standard deviations before and after optimization among the BOA method and the methods in the previous studies such as the Particle Swarm Optimization (PSO) algorithm, Simulated Annealing (SA) method, and Kuang. It was observed that the standard deviations are more accurate in most baselines in the case of BOA (Fig. 6). Moreover, in the BOA method, after decreasing the precision of the network by $(2.00 \mathrm{~mm})$, a total of 87 baselines were removed (saving $56.9 \%$ of the fieldwork), while PSO, SA, and Kuang methods saved $56.9 \%, 51.63 \%$, and $47.7 \%$ field work, respectively. The total lengths of removed baselines are $1382.85 \mathrm{Km}, 1322$ $\mathrm{Km}$, and $1136.33 \mathrm{Km}$ for the methods BOA, PSO, and SA, respectively.

\section{CONCLUSION}

This paper aims to shed the light on the importance of new technologies in selecting optimal baselines for GPS networks using the global optimization techniques. We used the Butterfly Optimization Algorithm (BOA) as a new artificial intelligence method for the choice of optimal baselines in GPS networks. The algorithm has been tested on GPS network, which can be solved using the traditional method of Kuang (1996). Our example illustrates that BOA can be adopted effectively to solve complex optimization problems since the number of the baselines removed under the influence of the BOA ( 87 baselines) was higher than the number of the baselines removed by 
the traditional method ( 73 baselines). Furthermore, the total length of deleted baselines reached $1382.85 \mathrm{~km}$. This result is one of the best results obtained using artificial intelligence methods. The results of this paper were more efficient than those of the traditional method by $19.2 \%$, where they were better than those of the artificial methods in terms of length, and it enhanced the SA method by $21.7 \%$ and PSO method by $4.6 \%$. As a result, the use of the BOA is effective and applicable.

\section{REFERENCES}

Alizadeh-Khameneh M.A. 2017, "Optimal Design in Geodetic GNSS Based Networks".

Amiri-Simkooei A. R. 2012, Asgari J., Zangeneh-Nejad F., and Zaminpardaz S., "Basic Concepts of Optimization and Design of Geodetic Networks," vol. 138, no. 4, pp. 172-183.

Arora S. and Singh S.2015, "Butterfly algorithm with l'evy flights for global optimization, in Signal Processing, Computing, and Control (2015 ISPCC)", pp. 220-224,2015 International Conference on, IEEE .

Arora S. and Singh S.2017, "An improved butterfly optimization algorithm with chaos," Journal of Intelligent \& Fuzzy Systems, vol. 32, no. 1, pp. 1079-1088.

Arora S. ,Singh S. and Yetilmezsoy,2018. "A modified butterfly optimization algorithm for mechanical design optimization problems", Journal of the Brazilian Society of Mechanical Sciences and Engineering , vol.40, no.1, pp. 1-17.

Arora S. and Singh S.2019, "Butterfly optimization algorithm: a novel approach for global optimization," Soft Comput., vol. 23, no. 3, pp. 715-734, 2019.

Baarda W., 1981. "S-Transformations and Criterion Matrices, Neth. Geod". Comm Pub. on Geodesy, New Series, 2nd Ed., Vol. 5, Delft, The Netherlands.

Doma M.I. and Elshoney A.F, 2011."A new method for designing the optimum geodetic networks using genetic algorithms," Journal of Engineering and Applied Science, vol.58, no.2, pp. 109-125.

Doma M. I.; Faried Y. A.; Sharkawi, A.A. and Azmi, M. T.2013, "A Methodology for Optimum Choice of Observed Baselines in GPS Networks Using Simulated Annealing Algorithm" Civil Engineering Research Magazine, Al-Azhar University, Vol. 35, No. 3.

Doma M. I. 2013,"Particle Swarm Optimization in Comparison with Classical Optimization for GPS Network Design", Journal of Geodetic Science vol. 3, no.4, pp. 250-257.

Doma M. I. and Sedeek A.2014, "Comparison of PSO, GAs and Analytical Techniques in second-order design of Deformation Monitoring Networks", Journal of Applied Geodesy,vol.8, no.1, pp. 21-30.

Dwivedi R. and Dikshit O. 2013, "A Comparison of Particle Swarm Optimization (PSO) and Genetic algorithm (GA) in Second-Order design (SOD) of GPS networks", Journal of Applied Geodesy vol. 7, 135-146.

Grafarend, E.W. 1974, "Optimization of geodetic networks." Bolletino di Geodesia a Science Affini, Volume 33, No. 4, pp. $351-406$.

Hoque Z. 2016, "Basic Concept of GPS and Its Applications", IOSR Journal Of Humanities And Social Science vol. 21, no. 3, pp. 31-37.

Habeeb, A. 2018 "Introduction to Artificial Intelligence ” University of Mansoura, no. June,.

Kuang S. L.1991, "Optimization and Design of Deformation Monitoring Schemes". Ph. D. dissertation, Department of Surveying Engineering Technical Report No. 157, University of New Brunswick, Fredericton, New Brunswick, Canada .

Kuang S. L.1996, "Geodetic Network Analysis and Optimal Design: Concept and Applications", Ann Arbor Press, Inc., Chelsea, Michigan. 
Kota, L. 2018 “ARTIFICIAL INTELLIGENCE IN LOGISTICS APPLICATIONS AND ALGORITHMS” Advanced Logistic Systems, Vol. 12, no. 1 ,pp. 47-60.

Postek.P.2021, "New Computer Simulation Method for First-Order Design of Geodetic Network : Aiming for Low Cost," vol. 147, no. 1985.

Yetkin M., Inal C., Yigit C. O.2008, "Optimal Design of Deformation Monitoring Networks Using PSO Algorithm", 13th FIG Symposium on Deformation Measurement and Analysis and 4th IAG Symposium on Geodesy for Geotechnical and Structural Engineering, LNEC, LISBON .

Yetkin M., Inal C. and Yigit C. O. 2009, "Use of the particle swarm optimization algorithm for second-order design of leveling networks," Journal of Applied Geodesy, vol. 3, no. 3, pp. 171-178.

Yetkin M., Inal C., Yigit C. O.2011, "The optimal design of baseline configuration in GPS networks by using the particle swarm optimization algorithm", Survey Review 43, 700-712. 